\title{
Publisher Correction: Fgf10 is essential for limb and lung formation
}

Keisuke Sekine, Hideyo Ohuchi, Masanori Fujiwara, Masahiro Yamasaki, Tatsuya Yoshizawa, Takashi Sato,

Naoko Yagishita, Daisuke Matsui, Yoshihiko Koga, Nobuyuki Itoh and Shigeaki Kato

Correction to: Nature Genetics https://doi.org/10.1038/5096, published online 1 January 1999.

In the version of the paper initially published, Fig. 5a was inadvertently duplicated and presented as both Figs. $5 \mathrm{a}$ and $5 \mathrm{f}$, and the correct image for Fig. $5 \mathrm{f}$ was omitted. Images of the original and corrected figure panels are shown in the correction notice.
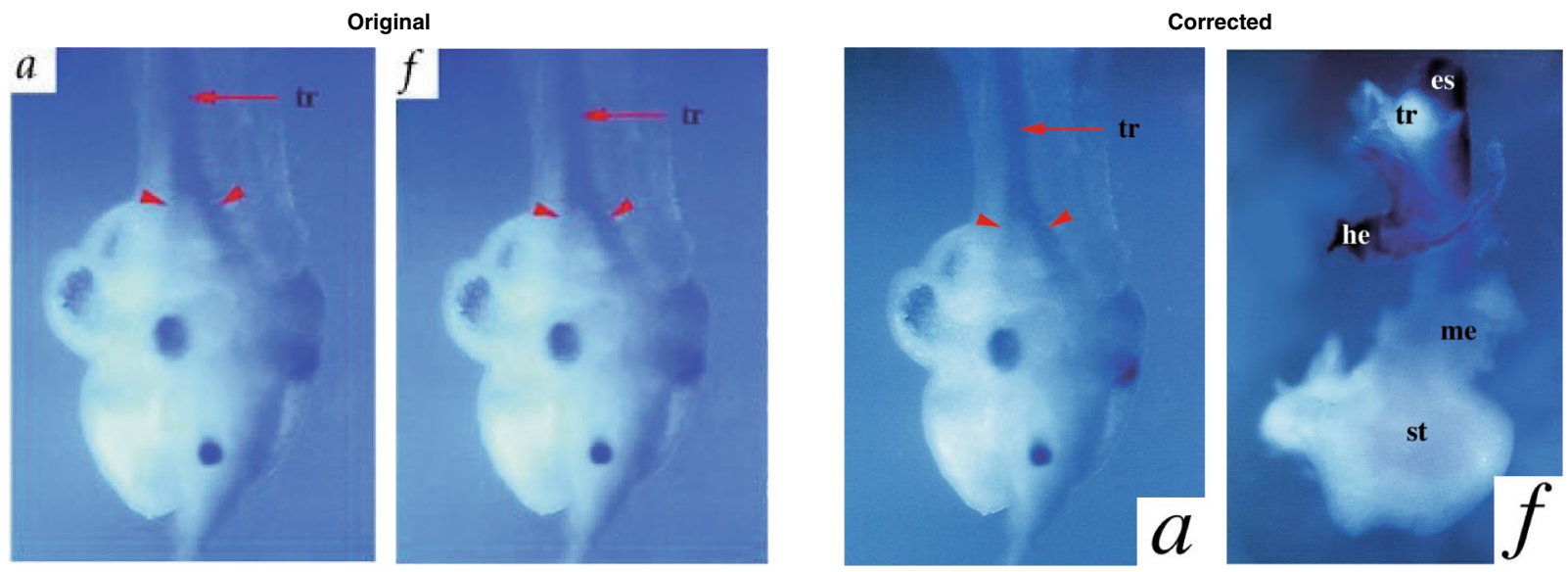

Fig. 5 | Original and corrected.

Published: 4 April 2019

https://doi.org/10.1038/s41588-019-0396-9 